\section{Ambiguous edge detection leads to subjective contours}

\section{S. J. DAVIS and M. E. JERNIGAN \\ Department of Systems Design, University of Waterloo Waterloo, Ontario N2L 3G1, Canada}

Many authors have argued that visual preprocessing alone cannot explain subjective contours. See, for example, Coren and Theodor (1975), Rock and Anson (1979), and Tyler (1977). However, none of the arguments precludes the possibility that a cognitive mechanism that produces the illusion acts in response to ambiguities that arise during preprocessing. We would like to argue that the brain "chooses" to perceive subjective contours in certain images in order to resolve a structural ambiguity created by the edge-detecting subsystem (EDS) of the human visual system.

In our model, we assume that a sudden change in the visual field produces a blurry initial response that becomes clearer as the visual field stabilizes. This idea grew out of speculation on Wilson and Bergen's (1979) study of transient and sustained mechanisms in the human visual system. However, instead of limiting our discussion to four mechanisms, we consider a continuum of transient and sustained response.

As in Wilson and Bergen, we use a single filter form to model both transient and sustained phenomena. However, we follow Marr (1976) and represent the EDS by a Gaussian smoothed Laplacian (GSL) form. Large values in the exponent of the Gaussian term cause severe blurring that corresponds to the transient behavior of the EDS. By allowing the magnitude of the exponent to decrease with time, the spatial resolution of the GSL improves until an output representative of the sustained response of the EDS is obtained.

Wilson and Bergen did not claim that the mechanisms they worked with were edge detectors. However, the stimuli they used consisted of simple edge information. By following the procedure described in Jernigan and Wardell (1981), it is possible to use GSL filters to model the Wilson and Bergen mechanisms to within the limits of experimental error. Therefore, we feel justified in treating the Wilson and Bergen mechanisms as a special case of the edgedetecting subsystem.

To simulate the operation of the EDS, we process digital images with discrete representations of the Gaussian smoothed Laplacian filters and record the location of output values that are greater than a prespecified magnitude. This process simulates the detection of edges with contrast above a minimum threshold. Details of the simulation are presented in Davis (Note 1).

Although we have assumed that the smoothing parameter in the GSL starts with some large initial value immediately following a saccade and then decreases with time until the next saccade, we only consider the output of the EDS at isolated points in time. We define the structural components of an image as neighboring picture elements with similar gray level (regions of constant intensity) and then consider what the EDS output implies about the structure of the input image.

The image of Figure 1 was filtered and thresholded using a filter corresponding to the Wilson and Bergen $\mathbf{N}$ mechanism (sustained response) to produce Figure 2. This "edge image" is similar to the image that would result if the input consisted of four right angles (Figure 3). Thus, one would have difficulty distinguishing Figures 1 and 3 on the basis of $\mathrm{N}$-mechanism filtering alone.

By perceiving subjective contours in Figure 1, the human visual system reduces the ambiguity concerning the way the output from the EDS may be interpreted. Consider an image similar to Figure 1 but with a central region that is brighter than the background. This image may be thought of as a "synthesized illusion" (i.e., it is what an observer perceives when viewing Figure 1). The result of an $\mathrm{N}$ mechanism filtering of such an image is shown in Figure 4. The new "edge image" is in close agreement with the structure of the synthesized illusion. Using standard image measures (e.g., sum of squared error), the distance between Figure 1 and the synthesized illusion is always less than the distance between Figures 1 and 3. Thus, perceiving a subjective contour in

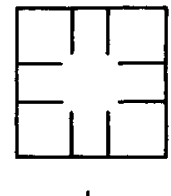

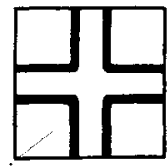

2

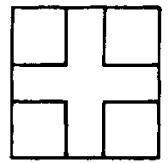

3

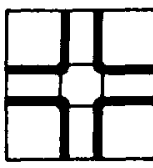

4

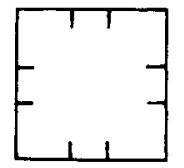

5
Figures 1-5. (1) Subjective contour illusion. (2) N-mechanism edge information contained in (1). (3) Second image with edge information similar to (2). (4) Edge information perceived in (1). (5) Figure 1 modified to make the illusion less compelling. 
Figure 1 is a simpler way to obtain a consistent EDS output than perceiving Figure 1 as Figure 3.

If the horizontal and vertical bars in Figure 1 are moved farther apart, as in Figure 5, the illusion becomes weaker. An ambiguous "edge image" similar to Figure 2 is only produced by Figure 5 during the early stages of processing (transient response). We believe the illusion appears weaker in Figure 5 than in Figure 1 because the EDS output is not ambiguous for the entire time an observer studies the former image, while it is ambiguous during the entire time he studies the latter. If our supposition is correct, it should be possible to make the illusion in Figure 5 stronger by increasing the percentage of viewing time during which the EDS output is ambiguous. One way to do this would be to have the observer move his head while viewing the image. A similar effect may explain why 4 of the 20 subjects who did not see a subjective contour with their heads held stationary during the first part of Experiment 4B in Rock and Anson (1979) did see the subjective contour when they were asked to move their heads back and forth.

\section{REFERENCE NOTE}

1. Davis, S. J. Visual preprocessing of a subjective contour illusion (Systems Design Workshop Report). Waterloo: Department of Systems Design, University of Waterloo, 1981.

\section{REFERENCES}

Coren, S., \& Theodor, L. H. Subjective contours: The inadequacy of brightness contrast as an explanation. Bulletin of the Psychonomic Society, 1975, 6, 87-89.

Jernigan, M. E., \& Wardell, R. W. Does the eye contain optimal edge detectors? IEEE Transactions on Systems, Man, and Cybernetics, 1981, SMC11, 441-444.

Marr, D. Early processing of visual information. Proceedings of the Royal Society of London $B, 1976,275,483-524$.

Rock, I., \& Anson, R. Illusory contours as the solution to a problem. Perception, 1979, 8, 665-681.

TyLE R, C. W. Is the illusory triangle physical or imaginary? Perception, 1977, 6, 603-604.

Wilson, H. R., \& Bergen, J. R. A four mechanism model for threshold spatial vision. Vision Research, 1979, 19, 19-32.

(Manuscript received September 21, 1981; accepted for publication September 24, 1981.) 